\title{
NUMERICAL COMPUTATION OF RANK-ONE CONVEX ENVELOPES *
}

\author{
GEORG DOLZMANN ${ }^{\dagger}$
}

\begin{abstract}
We describe an algorithm for the numerical computation of the rank-one convex
\end{abstract} envelope of a function $f: \mathbb{M}^{m \times n} \rightarrow \mathbb{R}$. We prove its convergence and an error estimate in $L^{\infty}$.

Key words. rank-one convexity, generalized convex envelopes, nonconvex variational problems.

AMS subject classifications. $65 \mathrm{~N} 12,65 \mathrm{~N} 15,65 \mathrm{~N} 30$

PII. S0036142997325581

1. Introduction. A real valued function $f$ defined on the space $\mathbb{M}^{m \times n}$ of all real $m \times n$ matrices is said to be rank-one convex if the functions $\varphi: \mathbb{R} \rightarrow \mathbb{R}$ defined by $\varphi(t)=f(F+t R)$ are convex $\forall F, R \in \mathbb{M}^{m \times n}$, rank $R \leq 1$. If a given function $f$ is not rank-one convex, how can one compute the rank-one convex envelope $f^{r c}$ of $f$, i.e., the largest function $f^{r c} \leq f$ that is rank-one convex? This is a difficult problem since, in general, the value of $f^{r c}$ for a given matrix $F$ depends on the values of $f$ on the whole space $\mathbb{M}^{m \times n}$ and not just on the values of $f$ on a bounded neighborhood of $F$.

The problem of computing the rank-one convex envelope of a function arises, for example, in the mathematical analysis of models in nonlinear elasticity or homogenization: The fundamental question here consists in finding a deformation $u: \Omega \subset$ $\mathbb{R}^{n} \rightarrow \mathbb{R}^{n}$ which minimizes an energy functional

$$
I(u)=\int_{\Omega} W(D u) \mathrm{dx}
$$

subject to suitable boundary conditions, e.g., $u(x)=F x$ on $\partial \Omega$. Here $\Omega$ is the reference configuration of an elastic body and $W \geq 0$ is a stored energy density. In general this problem has no solution (in appropriate Sobolev spaces) if $W$ is not quasiconvex. Here $W$ is said to be quasiconvex if for affine boundary conditions the homogeneous deformation is minimizing, i.e.,

$$
\int_{\Omega} W(F+D \varphi) d x \geq \int_{\Omega} W(F) d x \quad \forall F \in \mathbb{M}^{n \times n}, \forall \varphi \in C_{0}^{\infty}\left(\Omega ; \mathbb{R}^{n}\right)
$$

(see $[\mathrm{M}],[\mathrm{BJ}])$. The fundamental theorem in relaxation (see $[\mathrm{Da}]$ ) states that

$$
\min _{u \in \mathcal{A}} \int_{\Omega} W^{q c}(D u) d x=\inf _{u \in \mathcal{A}} \int_{\Omega} W(D u) d x,
$$

where $\mathcal{A}$ is a suitable class of functions, e.g., $\mathcal{A}=\left\{u \in W^{1, \infty}\left(\Omega ; \mathbb{R}^{n}\right): u(x)=\right.$ $F x$ on $\partial \Omega\}$. Here $W^{q c}$ is the quasiconvex envelope of $W$, i.e., the largest quasiconvex minorant of $W$. Therefore we can compute the relaxed or effective energy by

* Received by the editors August 6, 1997; accepted for publication (in revised form) October 28, 1998; published electronically September 8, 1999. This work was partially supported by ARO and NSF through grants to the Center for Nonlinear Analysis, Carnegie Mellon University, Pittsburgh, PA.

http://www.siam.org/journals/sinum/36-5/32558.html

†California Institute of Technology, Pasadena, CA 91125 (georg@mis.mpg.de). The work of the author was performed while on leave from Max Planck Institute for Mathematics in the Sciences, Inselstrasse 22-26, D-04103 Leipzig, Germany. 
minimizing the relaxed functional where one replaces $W$ by $W^{q c}$. However, $W^{q c}$ is difficult to compute and analytically only known in very special cases where $W^{q c}$ and $W^{r c}$ coincide (see, e.g., [KS2], [Pi], [LDR]). It is unknown whether this is always true if, for example, the function $W$ has additional symmetries like those implied by frame indifference. Since quasiconvexity implies rank-one convexity (the converse is not true; see [Sv2]) we have $W^{q c} \leq W^{r c} \leq W$ and hence the equalities

$$
\min _{u \in \mathcal{A}} \int_{\Omega} W^{q c}(D u) d x=\inf _{u \in \mathcal{A}} \int_{\Omega} W^{r c}(D u) d x=\inf _{u \in \mathcal{A}} \int_{\Omega} W(D u) d x .
$$

Numerical schemes for the computation of the relaxed energy based on these identities require approximations of $W^{r c}$ or $W^{q c}$. This is an extremely complex task since in the cases of physical interest $(n=m=2$ and $n=m=3$ ) these functions are defined on four- and nine-dimensional matrix spaces, respectively. There are no reasonable numerical schemes for the computation of the quasiconvex envelope known. Here we describe a reliable algorithm for the computation of $W^{r c}$ restricted to a grid in $\mathbb{M}^{m \times n}$ and give a rigorous convergence proof. The numerical examples in section 5 show even better convergence properties than those predicted analytically. Unfortunately it is unknown whether the discrete function can be extended from the grid to the whole space preserving suitable convexity properties; see section 3 for a short discussion of this issue. Thus it remains a challenging problem to combine the numerical rank-one convexification with the original minimization problem. For numerical methods to treat the nonconvex problem see the recent survey article [L].

A closely related problem concerns the computation of certain generalized convex hulls of sets $K \subset \mathbb{M}^{m \times n}$. Define the quasiconvex hull $K^{q c}$ of a given set $K$ by

$$
K^{q c}=\left\{X \in \mathbb{M}^{m \times n}, f(X) \leq \sup _{Y \in K} f(Y) \quad \forall f: \mathbb{M}^{m \times n} \rightarrow \mathbb{R} \text { quasiconvex }\right\}
$$

(see [Sv3]); we define the rank-one convex hull $K^{r c}$ and the separately convex hull $K^{s c}$ of $K$ analogously. Here we say that $f$ is separately convex if it is convex in each variable. If $K=\{X, W(X)=0\}$, then $K^{q c}$ is closely related to the set of (affine) boundary conditions for the variational problem above for which there exist Lipschitz continuous functions $u$ with $I(u)=0$ (see [DM], [MS]). Again it is, in general, very difficult to compute the quasiconvex hull of a given set explicitly (see, e.g., [BJ], [Sv3]). Since $K^{r c} \subset K^{q c}$, the rank-one convex hull gives an inner bound for the quasiconvex hull. If $K$ is compact, then $K^{r c}=\left\{X,(\operatorname{dist}(\cdot, K))^{r c}(X)=0\right\}$. For further generalization of notions of convexity and convex hulls as well as their properties see, for example, [MP], [T], and [Z].

In this paper we propose an algorithm $\mathcal{A}$ that computes a discrete rank-one convex envelope $f^{h}$ of $f$ on a given uniform grid $\mathcal{G}_{h} \subset \mathbb{M}^{m \times n}$. If $f$ is bounded from below by a rank-one convex function, we prove that the functions $f^{h}$ converge to $f^{r c}$ in the grid points as the mesh size $h$ tends to zero. The algorithm is based on the idea that it is possible to compute $f^{r c}$ by successively rank-one convexifying $f$ on all rank-one lines in $\mathbb{M}^{m \times n}$ (see $[\mathrm{KS} 2$, Section $5 \mathrm{C}]$ for a discussion of this and related ideas). One problem in numerical schemes based on this approach is that given two matrices in the grid it is difficult to decide whether they lie on a rank-one line or not. This is due to the fact that the set $\{\operatorname{det} X=0\}$ is not open in $\mathbb{M}^{m \times n}$ and thus not stable with respect to perturbations. We solve this problem by defining a suitable set $\mathcal{R}_{h}$ of rank-one matrices with the property that $F_{h}+R_{h} \in \mathcal{G}_{h}$ for $F_{h} \in \mathcal{G}_{h}$ and $R_{h} \in \mathcal{R}_{h}$. It is crucial for the proof of the convergence of the algorithm that the numerical 
scheme performs one-dimensional convexifications only on rank-one lines and not on "approximate" rank-one lines defined by a condition of the form $|\operatorname{det} R|<\tau$, where $\tau$ is a given tolerance. A closely related approach was independently analyzed in [Pl].

The paper is organized as follows: In section 2 we introduce the relevant notation and recall important facts concerning rank-one convexity. In section 3 we define the algorithm and state the convergence theorems. The proofs of the theorems are contained in section 4. We present some numerical examples in section 5 .

2. Preliminaries. In this section we recall basic facts concerning rank-one convexity and rank-one convex envelopes of functions $f: \mathbb{M}^{m \times n} \rightarrow \mathbb{R}$.

DEFINITION 2.1.

(i) A function $f: \mathbb{M}^{m \times n} \rightarrow \mathbb{R}$ is said to be rank-one convex if

$$
f\left(\lambda F_{1}+(1-\lambda) F_{2}\right) \leq \lambda f\left(F_{1}\right)+(1-\lambda) f\left(F_{2}\right)
$$

$\forall F_{1}, F_{2} \in \mathbb{M}^{m \times n}, \operatorname{rank}\left(F_{1}-F_{2}\right) \leq 1$ and $\lambda \in[0,1]$.

(ii) The rank-one convex envelope $f^{r c}$ of a function $f: \mathbb{M}^{m \times n} \rightarrow \mathbb{R}$ is defined by

$$
f^{r c}=\sup \{g \leq f, g \text { rank-one convex }\} .
$$

An equivalent definition of rank-one convexity can be given using the following property of matrices [Da, Section 4.1.1.3]. The pairs $\left(\lambda_{i}, F_{i}\right) \in \mathbb{R} \times \mathbb{M}^{m \times n}, i=$ $1, \ldots, N$, satisfy the condition $\mathcal{H}_{N}$ (we write $\left.\left(\lambda_{i}, F_{i}\right) \in \mathcal{H}_{N}\right)$ if the following holds: $\lambda_{i} \geq 0, \sum_{i=1}^{N} \lambda_{i}=1$, and

(i) $N=1: \mathcal{H}_{1}$ is always true;

(ii) if $N \geq 2$, then, up to a permutation, $\operatorname{rank}\left(F_{1}-F_{2}\right) \leq 1$ and if

$$
\begin{aligned}
\mu_{1}=\lambda_{1}+\lambda_{2}, G_{1}^{N-1} & =\frac{\lambda_{1} F_{1}+\lambda_{2} F_{2}}{\lambda_{1}+\lambda_{2}}, \\
\mu_{i}=\lambda_{i+1}, G_{i}^{N-1} & =F_{i+1}, i=2, \ldots, N-1,
\end{aligned}
$$

then $\left(\mu_{i}, G_{i}^{N-1}\right) \in \mathcal{H}_{N-1}$. It is convenient to define $G_{i}^{N}=F_{i}, i=1, \ldots, N$. Applying this definition recursively defines matrices $H_{i}=G_{1}^{N-i}, i=1, \ldots, N-1$. Clearly $H_{N-1}=\sum_{i=1}^{N} \lambda_{i} F_{i}$. The geometric interpretation of the definition above is given by a graph $\mathcal{G}\left(\lambda_{i}, F_{i}\right)$ with leaves $F_{i}$, inner nodes $H_{i}$, and edges that are rank-one lines in $\mathbb{M}^{m \times n}$; see Figure 2.1. Conversely, any graph of this form defines a set of pairs $\left(\lambda_{i}, F_{i}\right)$ with the properties above. Removing the edge that contains $H_{N-1}$ as an inner point one obtains two disjoint trees with the following properties.

Remark 2.1. If $\left(\lambda_{i}, F_{i}\right) \in \mathcal{H}_{N}$ with $N>2$, then there exists $k \in\{1, \ldots, N-1\}$ such that, up to a permutation,

$$
\left(\frac{\lambda_{i}}{\lambda_{1}+\cdots+\lambda_{k}}, F_{i}\right)_{i=1, \ldots, k} \in \mathcal{H}_{k}, \quad\left(\frac{\lambda_{i}}{\lambda_{k+1}+\cdots+\lambda_{N}}, F_{i}\right)_{i=k+1, \ldots, N} \in \mathcal{H}_{N-k}
$$

and if

$$
\mu_{1}=\lambda_{1}+\cdots+\lambda_{k}, \quad \mu_{2}=\lambda_{k+1}+\cdots+\lambda_{N}
$$

and

$$
G_{1}=\frac{\lambda_{1} F_{1}+\cdots+\lambda_{k} F_{k}}{\lambda_{1}+\cdots+\lambda_{k}}, \quad G_{2}=\frac{\lambda_{k+1} F_{k+1}+\cdots+\lambda_{N} F_{N}}{\lambda_{k+1}+\cdots+\lambda_{N}},
$$

then $\left(\mu_{i}, G_{i}\right) \in \mathcal{H}_{2}$. 

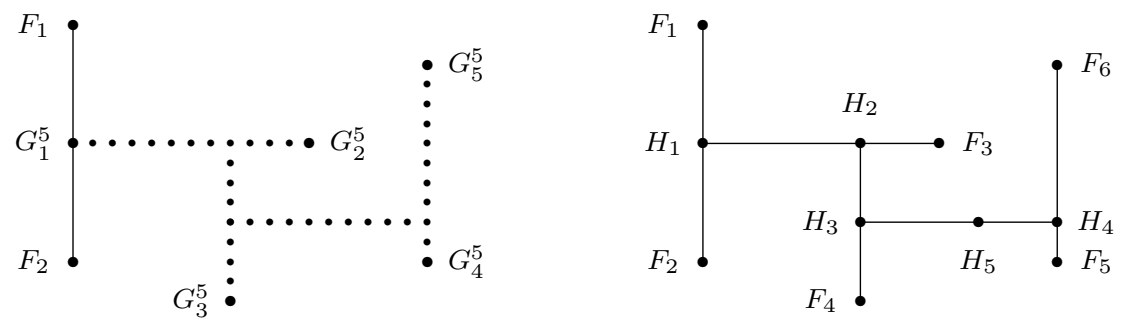

FIG. 2.1. Generation of the graph $\mathcal{G}\left(\lambda_{i}, F_{i}\right), N=6$. The solid and the dotted lines represent rank-one lines.

Lemma 2.2 (see [Da, Proposition 4.1.4, Theorem 5.1.1]).

(i) A function $f: \mathbb{M}^{m \times n} \rightarrow \mathbb{R}$ is rank-one convex if and only if

$$
f\left(\sum_{i=1}^{N} \lambda_{i} F_{i}\right) \leq \sum_{i=1}^{N} \lambda_{i} f\left(F_{i}\right) \quad \forall\left(\lambda_{i}, F_{i}\right) \in \mathcal{H}_{N}, \forall N .
$$

(ii) The rank-one convex envelope $f^{r c}$ is given by

$$
f^{r c}(F)=\inf \left\{\sum_{i=1}^{N} \lambda_{i} f\left(F_{i}\right),\left(\lambda_{i}, F_{i}\right) \in \mathcal{H}_{N} \text { and } F=\sum_{i=1}^{N} \lambda_{i} F_{i}\right\} .
$$

It is not possible to bound the number $N$ in the definition of $f^{r c}$. We denote by $\mathcal{E}^{N}(K)$ the set of all functions for which the rank-one convex envelope can be computed using no more than $N$ matrices $F_{i} \in K$.

The next two lemmas show that it is sufficient to use matrices $F_{i} \in B_{r}(0) \subset \mathbb{M}^{m \times n}$ in the formula for $f^{r c}$ in Lemma 2.2 if $f$ is rank-one convex outside of $B_{r}(0)=\{F \in$ $\left.\mathbb{M}^{m \times n}:|F| \leq r\right\}$ and satisfies an appropriate lower bound on $B_{r}(0)$. This is in the spirit of results in [Pe1, Lemma 6.3], [Pe2, Section 4], and [Sv1, Lemma 3.1].

Lemma 2.3. Assume that $f, g^{r c}: \mathbb{M}^{m \times n} \rightarrow \mathbb{R}$ are continuous, $g^{r c}$ is rank-one convex, $f \geq g^{r c}$ on $\mathbb{M}^{m \times n}, f=g^{r c}$ on $\mathbb{M}^{m \times n} \backslash B_{r}(0)$, and that $f$ is rank-one convex on $B_{r}(0)$. Then $f$ is rank-one convex.

Proof. It suffices to consider rank-one lines that intersect $\partial B_{r}(0)$. Let $F_{1} \in B_{r}(0)$, $F_{2} \in \mathbb{M}^{m \times n} \backslash B_{r}(0)$, with $\operatorname{rank}\left(F_{1}-F_{2}\right) \leq 1, \lambda \in[0,1]$ and $\bar{F}=\lambda F_{1}+(1-\lambda) F_{2}$. If $\bar{F} \in \mathbb{M}^{m \times n} \backslash B_{r}(0)$, then

$$
\begin{aligned}
f(\bar{F}) & =g^{r c}(\bar{F}) \leq \lambda g^{r c}\left(F_{1}\right)+(1-\lambda) g^{r c}\left(F_{2}\right) \\
& \leq \lambda f\left(F_{1}\right)+(1-\lambda) f\left(F_{2}\right) .
\end{aligned}
$$

If $\bar{F} \in B_{r}(0)$, define $F_{0}=\nu F_{1}+(1-\nu) F_{2} \in \partial B_{r}(0)$ and $\mu$ by $\bar{F}=\mu F_{1}+(1-\mu) F_{0}$. We obtain

$$
\begin{aligned}
f(\bar{F}) & =f\left(\mu F_{1}+(1-\mu) F_{0}\right) \\
& \leq \mu f\left(F_{1}\right)+(1-\mu) f\left(F_{0}\right) \\
& \leq(\mu+(1-\mu) \nu) f\left(F_{1}\right)+(1-\mu)(1-\nu) f\left(F_{2}\right) .
\end{aligned}
$$

Since $(\mu+(1-\mu) \nu) F_{1}+(1-\mu)(1-\nu) F_{2}=\bar{F}$ we have $\mu+(1-\mu) \nu=\lambda$ and the result follows. The case that $F_{1}, F_{2} \in \mathbb{M}^{m \times n} \backslash B_{r}(0)$ and $\bar{F} \in B_{r}(0)$ can be handled similarly. 
Lemma 2.4. Assume that $f, g^{r c}: \mathbb{M}^{m \times n} \rightarrow \mathbb{R}$ are continuous, $f \geq g^{r c}$ on $\mathbb{M}^{m \times n}$, $f=g^{r c}$ on $\mathbb{M}^{m \times n} \backslash B_{r}(0)$ and that $g^{r c}$ is rank-one convex. Define $\tilde{f}$ by $\tilde{f}(F)=f(F)$ if $F \in \mathbb{M}^{m \times n} \backslash B_{r}(0)$ and by

$$
\tilde{f}(F)=\inf \left\{\sum_{i=1}^{N} \lambda_{i} f\left(F_{i}\right),\left(\lambda_{i}, F_{i}\right) \in \mathcal{H}_{N}, F_{i} \in B_{r}(0), F=\sum_{i=1}^{N} \lambda_{i} F_{i}\right\}
$$

for $F \in B_{r}(0)$. Then $\tilde{f}=f^{r c}$.

Proof. We first show that $\tilde{f}$ and $g^{r c}$ satisfy the assumptions of Lemma 2.3. Clearly $\tilde{f} \geq g^{r c}$. Using the same arguments as in the proof of Theorem 5.1.1 in [Da] it is easy to see that $\tilde{f}$ is rank-one convex on $B_{r}(0)$ and hence continuous on $B_{r}(0)$. We finally check that $\tilde{f}$ is continuous on $\partial B_{r}(0)$. To see this fix $F_{0} \in \partial B_{r}(0), \varepsilon>0$ and choose $\delta>0$ such that $\left|f(F)-f\left(F_{0}\right)\right|<\varepsilon$ if $\left|F-F_{0}\right|<\delta$. If $G \in B_{\delta}\left(F_{0}\right) \cap B_{r}(0)$, then

$$
g^{r c}(G) \leq \tilde{f}(G) \leq f(G) \leq g^{r c}\left(F_{0}\right)+\varepsilon .
$$

Thus $\tilde{f}(G) \rightarrow f\left(F_{0}\right)$ as $G \rightarrow F_{0}$.

It remains to show that $\tilde{f}=f^{r c}$. By Lemma 2.3 the function $\tilde{f}$ is rank-one convex and hence $\tilde{f} \leq f^{r c}$. On the other hand, the formula for $f^{r c}$ in Lemma 2.2 for $f^{r c}$ shows that $f^{r c} \leq \tilde{f}$. This proves $\tilde{f}=f^{r c}$.

3. Discretization and description of the algorithm. In this section we describe the algorithm for the computation of the rank-one convex envelope of a given function $f$ on a ball $B_{r}(0) \subset \mathbb{M}^{m \times n}$. For $0<h<1$ and $r>1$ denote by $\mathcal{G}(h, r)$ the uniform grid

$$
\mathcal{G}(h, r)=\left\{h G: G \in \mathbb{M}^{m \times n}, G_{i j} \in \mathbb{Z}, i=1, \ldots, m, j=1, \ldots, n\right\} \cap B_{r}(0) .
$$

For each $h$ define a discrete set of rank-one directions $\mathcal{R}_{h, r}$ by

$$
\mathcal{R}_{h, r}=\left\{h R: R=a \otimes b, a \in \mathbb{Z}^{m}, b \in \mathbb{Z}^{n}, \sqrt{h}|a|, \sqrt{h}|b| \leq \sqrt{2 r}\right\} .
$$

Finally denote by $\ell_{h, r}(F, R), \operatorname{rank}(R)=1$, the intersection of the grid $\mathcal{G}(h, r)$ with the rank-one line $F+t R$,

$$
\ell_{h, r}(F, R)=\{F+t R, t \in \mathbb{R}\} \cap \mathcal{G}(h, r)
$$

and by $f^{h}(F, R)(\cdot)$ the restriction of $f^{h}$ to $\ell_{h, r}(F, R)$ (which can now be considered as a function of one variable given at finitely many points). A function $f^{h}$ is called rank-one convex on $\mathcal{G}(h, r)$ if the piecewise affine interpolation $P_{1} f^{h}(F, R)$ of $f^{h}(F, R)$ is convex for all rank-one lines $\ell_{h, r}(F, R)$. It seems to be unknown whether one can extend a function $f^{h}$ which is rank-one convex on $\mathcal{G}(h, r)$ to a rank-one convex function on $B_{r}(0)$. However, it is easy to see that simple multilinear interpolation does not work. Consider, for example, the convex function $f(X)=\left(-X_{11}-X_{12}-X_{21}-X_{22}+1\right)^{+}$, where $a^{+}=\max \{a, 0\}$. Let $f^{h}$ be its restriction to $\mathcal{G}(1, \infty)$. Since $f^{h}$ vanishes at all corners of the four-dimensional unit cube except for zero, the multilinear interpolation of $f^{h}$ on $[0,1]^{4}$ is given by $M(X)=\left(X_{11}-1\right)\left(X_{12}-1\right)\left(X_{21}-1\right)\left(X_{22}-1\right)$. Consider now

$$
m(t)=M\left(\left(\begin{array}{ll}
1 & 0 \\
1 & 0
\end{array}\right)+t\left(\begin{array}{ll}
-1 & 1 \\
-1 & 1
\end{array}\right)\right)=M\left(\left(\begin{array}{ll}
1-t & t \\
1-t & t
\end{array}\right)\right) .
$$

Since $m(t)=t^{2}(1-t)^{2}$ fails to be convex on $[0,1]$ we conclude that $M$ is not rank-one convex.

We are now in a position to describe the algorithm $\mathcal{A}$ as follows. 


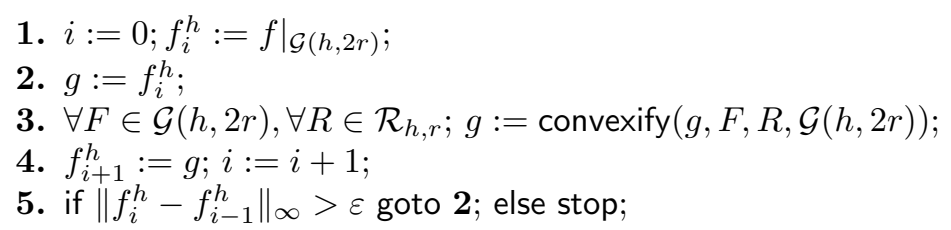

In the description of the algorithm $\mathcal{A}$ we use a subroutine convexify $(g, F, R, \mathcal{G}(h, 2 r))$ that computes the convexification of $g$ restricted to $\ell_{h, 2 r}(F, R)$ (see, e.g., [B, Co]). The parameter $\varepsilon$ is a given precision. The following two theorems describe the properties of the algorithm $\mathcal{A}$.

THEOREM 3.1. Assume that $f \geq g^{r c}$ on $B_{r}(0)$ for some rank-one convex function $g^{r c}$. Then there exists a rank-one convex function $f^{h}: \mathcal{G}(h, r) \rightarrow \mathbb{R}$ such that the functions $f_{i}^{h}$ defined in $\mathcal{A}$ converge to $f^{h}$.

In the following theorem we consider a sequence $\mathcal{G}\left(h_{i}, r\right)$ of uniform grids for $h_{i} \rightarrow 0$. We define

$$
q_{i}=\frac{h_{i+1}}{h_{i}}, \quad \delta_{i}=\sqrt{m+n} \sqrt{2 r} \sqrt{h_{i}}
$$

and

$$
q\left(h_{0}, \ldots, h_{N-1}\right)=\max _{0 \leq i \leq N-2} q_{i}, \quad \delta\left(h_{0}, \ldots, h_{N-1}\right)=\sum_{i=0}^{N-2}\left(\delta_{i}+2 r q_{i}\right)
$$

ThEOREM 3.2. Assume that $f, g^{r c}: \mathbb{M}^{m \times n} \rightarrow \mathbb{R}$ are continuous, that $g^{r c}$ is rank-one convex, $f \geq g^{r c}$ on $\mathbb{M}^{m \times n}$, and $f=g^{r c}$ on $\mathbb{M}^{m \times n} \backslash B_{r}(0)$. Let $\mathcal{G}\left(h_{i}, r\right)$ be a sequence of uniform grids for $h_{i} \searrow 0$ such that $\mathcal{G}\left(h_{i}, \infty\right) \subset \mathcal{G}\left(h_{j}, \infty\right)$ if $j \geq i$. Then $f^{h_{i}}=\mathcal{A}\left(f, \mathcal{G}\left(h_{i}, 2 r\right)\right)$ converges to $f^{r c}$ in the following sense:

$$
\lim _{i \rightarrow \infty}\left\|f^{h_{i}}-f^{r c}\right\|_{\infty ; \mathcal{G}\left(h_{j}, r\right)}=0 \quad \forall j \in \mathbb{N} .
$$

Moreover, if $f \in \mathcal{E}^{N}\left(B_{r}(0)\right)$ is locally Lipschitz continuous and $L$ is a Lipschitz constant for $f$ on $B_{2 r}(0)$, then the following estimate holds for $\ell \geq k_{0}+N-1$ and any choice of indices $k_{0} \leq i_{0}<\cdots<i_{N-1} \leq \ell$ :

$$
\left\|f^{h_{\ell}}-f^{r c}\right\|_{\infty ; \mathcal{G}\left(h_{k_{0}}, r\right)} \leq L \delta\left(h_{i_{0}}, \ldots, h_{i_{N-1}}\right)+\left(\sup _{B_{2 r}(0)}|f|\right) q\left(h_{i_{0}}, \ldots, h_{i_{N-1}}\right) .
$$

An immediate consequence is the following corollary.

Corollary 3.3. Assume that $h_{k}=2^{-k}$ and that the hypotheses in Theorem 3.2 hold. Suppose in addition that $k_{0}$ is even and that $\delta\left(h_{k_{0}}, \ldots, h_{k_{0}+N-1}\right)<r$. Then the following estimate holds for $\ell \geq \frac{N+1}{2} k_{0}$ :

$$
\left\|f^{h_{\ell}}-f^{r c}\right\|_{\infty ; \mathcal{G}\left(h_{k_{0}}, r\right)} \leq\left(\sqrt{m+n} \sqrt{2 r} L+2 r \sup _{B_{2 r}(0)}|f|\right)(N-1) \sqrt{h_{k_{0}}} .
$$

Proof. Choose $i_{\ell}=\left(1+\frac{\ell}{2}\right) k_{0}$ in Theorem 3.2.

We postpone the proofs of the theorems to section 4 and conclude this section with some remarks concerning generalizations and the necessity of the assumptions in the theorems.

Remarks.

(1) The lower bound for $f$ is needed in order to avoid $f_{i}^{h} \searrow-\infty$ in the algorithm. 
(2) The assumption $f=g^{r c}$ outside $B_{r}(0)$ can be replaced by the assumption that $f^{r c}$ restricted to $B_{r}(0)$ does not depend on the values of $f$ outside $B_{r}(0)$. There are nontrivial examples of functions which are not rank-one convex outside $B_{r}(0)$, for which the rank-one convex envelope can be computed on $B_{r}(0)$ with the values of $f$ on $B_{r}(0)$ only; see section 5 for an example. The lower bound $f \geq g^{r c}$ for some rank-one convex function $g^{r c}$ is not too restrictive; there exist rank-one convex functions with linear growth at infinity (see [Sv1]).

(3) The fact that $f^{r c}$ can be computed locally is crucial for the convergence in Theorem 3.2. If, e.g.,

$$
g^{r c}(t)=k e^{-t^{2}}\left(\chi_{(-\infty,-r)}(t)+\chi_{(r, \infty)}(t)\right)+a(r) t^{2} \chi_{(-r, r)}(t)
$$

with $a(r)=k e^{-r^{2}} / r^{2}$ and $k>0$ then the rank-one convexification of $f$ on $(-r, r)$ can be any rank-one convex function $h$ with $a(r) r^{2} \geq h(t) \geq a(r) t^{2}$, but $f^{r c} \equiv 0$.

(4) A uniform estimate $\left\|f^{h_{i}}-f^{r c}\right\|_{\infty ; \mathcal{G}\left(h_{i}, r\right)} \leq C h_{i}^{1 / 3}$ has recently been obtained in $[\mathrm{DW}]$.

4. Convergence of the algorithm. In this section we prove the theorems stated in section 3 .

Proof of Theorem 3.1. We first show that $f_{i}^{h} \geq f_{i+1}^{h} \geq g^{r c}$. Clearly $f_{0}^{h} \geq g^{r c}$ and $f_{i}^{h} \geq f_{i+1}^{h}$. To prove that $f_{i+1}^{h} \geq g^{r c}$, it suffices to show that

$$
\tilde{w}:=\operatorname{convexify}(w, F, R, \mathcal{G}(h, 2 r)) \geq g^{r c} \quad \text { if } w \geq g^{r c}, \operatorname{rank} R \leq 1 .
$$

By the rank-one convexity of $g^{r c}$ we have $P_{1}\left(\left.w\right|_{\ell_{h, 2 r}(F, R)}\right) \geq P_{1}\left(\left.g^{r c}\right|_{\ell_{h, 2 r}(F, R)}\right)$ and the inequality follows directly from the definition of the rank-one convex envelope since $P_{1}\left(\left.g^{r c}\right|_{\ell_{h, 2 r}(F, R)}\right)$ is convex. Thus $f_{i}^{h}$ is a monotone decreasing sequence on $\mathcal{G}(h, 2 r)$ bounded from below and converges pointwise to a function $f^{h}$. It remains to show that $f^{h}$ is rank-one convex. Assume otherwise. Then there exist matrices $F_{1}, F_{2}, \bar{F} \in \mathcal{G}(h, r)$ such that $\operatorname{rank}\left(F_{1}-F_{2}\right) \leq 1, \bar{F}=\lambda F_{1}+(1-\lambda) F_{2}, \lambda \in[0,1]$, and

$$
f^{h}(\bar{F})=\lambda f^{h}\left(F_{1}\right)+(1-\lambda) f^{h}\left(F_{2}\right)+\delta, \quad \delta>0 .
$$

Choose $i$ large enough such that $f_{i}^{h}(X)-f^{h}(X)<\delta / 3$ for $X \in\left\{F_{1}, F_{2}, \bar{F}\right\}$. This implies

$$
f_{i}^{h}(\bar{F}) \geq f^{h}(\bar{F}) \geq \lambda f_{i}^{h}\left(F_{1}\right)+(1-\lambda) f_{i}^{h}\left(F_{2}\right)+\frac{2}{3} \delta .
$$

The computation of $f_{i+1}^{h}$ includes the convexification of $f_{i}^{h}$ on $\ell_{h, r}\left(\bar{F}, F_{1}-F_{2}\right)$ and thus decreases the value of $f_{i}^{h}(\bar{F})$ by at least $\frac{2}{3} \delta$. Consequently,

$$
f^{h}(\bar{F}) \leq f_{i+1}^{h}(\bar{F}) \leq f_{i}^{h}(\bar{F})-\frac{2}{3} \delta \leq f^{h}(\bar{F})-\frac{\delta}{3},
$$

a contradiction.

The proof of Theorem 3.2 relies on the following two lemmas, which allow us to construct for a given rank-one tree in $\mathbb{M}^{m \times n}$ a rank-one tree supported on the grid which is close to the given one.

Lemma 4.1. Let $h_{i}$ be a sequence as in Theorem 3.2. Assume that $F_{1}, F_{2} \in$ $\mathbb{M}^{m \times n}, \operatorname{rank}\left(F_{1}-F_{2}\right)=1,\left|F_{1}\right|,\left|F_{2}\right|<r, \bar{F}=\lambda F_{1}+(1-\lambda) F_{2}$ and that $\bar{F}^{h} \in \mathcal{G}\left(h_{j}, r\right)$ 
such that $\left|\bar{F}-\bar{F}^{h}\right|<\delta$. Then, for $\ell \geq j$ big enough, there exist $F_{1}^{h}, F_{2}^{h} \in \mathcal{G}\left(h_{\ell}, r+3 \delta\right)$, $\operatorname{rank}\left(F_{1}^{h}-F_{2}^{h}\right) \leq 1$, and $\lambda^{h} \in[0,1]$ such that

$$
\left|F_{1}-F_{1}^{h}\right|<3 \delta, \quad\left|F_{2}-F_{2}^{h}\right|<3 \delta, \quad\left|\lambda-\lambda^{h}\right|<\delta
$$

and $\bar{F}^{h}=\lambda^{h} F_{1}^{h}+\left(1-\lambda^{h}\right) F_{2}^{h}$.

Proof. By assumption we may choose $a \in \mathbb{R}^{m}, b \in \mathbb{R}^{n}$ such that $F_{1}-F_{2}=a \otimes b$. Replacing $a$ by $\tilde{a}=(|b| /|a|)^{1 / 2} a$ and $b$ by $\tilde{b}=(|a| /|b|)^{1 / 2} b$ if necessary, we may assume that $|a|=|b|$. Since $|a \otimes b|=|a||b|=\left|F_{1}-F_{2}\right| \leq 2 r$ we deduce $|a|,|b| \leq \sqrt{2 r}$. For $k>0$ ( $k$ will be chosen later) choose $a^{k} \in \sqrt{h_{k}} \mathbb{Z}^{m}, b^{k} \in \sqrt{h_{k}} \mathbb{Z}^{n}$, such that $\left|a^{k}\right| \leq|a|$, $\left|b^{k}\right| \leq|b|$, and

$$
\left|a^{k}-a\right| \leq \sqrt{m h_{k}}, \quad\left|b^{k}-b\right| \leq \sqrt{n h_{k}} .
$$

Clearly

$$
\left|a \otimes b-a^{k} \otimes b^{k}\right| \leq\left|a-a^{k}\right||b|+\left|b-b^{k}\right|\left|a^{k}\right| \leq \sqrt{m+n} \sqrt{2 r} \sqrt{h_{k}} .
$$

For $\ell \geq k$ let $p=\frac{h_{k}}{h_{\ell}}$. Since $\mathcal{G}\left(h_{k}, \infty\right) \subset \mathcal{G}\left(h_{\ell}, \infty\right)$ it follows that $p \in \mathbb{N}$. For $\delta_{\lambda}>0$ given $\left(\delta_{\lambda}\right.$ will be chosen later) we may choose $\ell$ big enough such that there exists $q \in \mathbb{N}$ with $\left|\lambda-\frac{q}{p}\right|<\delta_{\lambda}$. Let $\lambda^{\ell}=\frac{q}{p}$ and define $F_{1}^{\ell}$ and $F_{2}^{\ell}$ by

$$
\begin{aligned}
& F_{1}^{\ell}=\bar{F}^{h}+\left(1-\lambda^{\ell}\right)\left(a^{k} \otimes b^{k}\right), \\
& F_{2}^{\ell}=\bar{F}^{h}-\lambda^{\ell} a^{k} \otimes b^{k} .
\end{aligned}
$$

By construction $F_{1}^{\ell}, F_{2}^{\ell} \in \mathcal{G}\left(h_{\ell}, r\right)$,

$$
\begin{aligned}
\left|F_{1}^{\ell}-F_{1}\right| & \leq\left|\bar{F}-\bar{F}^{h}\right|+\left|1-\lambda^{\ell}\right|\left|a^{k} \otimes b^{k}-a \otimes b\right|+\left|\lambda-\lambda^{\ell}\right||a \otimes b| \\
& \leq \delta+\sqrt{m+n} \sqrt{2 r} \sqrt{h_{k}}+2 r \delta_{\lambda}
\end{aligned}
$$

and similarly $\left|F_{2}^{\ell}-F_{2}\right| \leq \delta+\sqrt{m+n} \sqrt{2 r} \sqrt{h_{k}}+2 r \delta_{\lambda}$. Now choose $k$ and $\ell, k \leq \ell$, big enough such that $\left|\lambda-\lambda^{\ell}\right| \leq \delta_{\lambda}:=\frac{\delta}{2 r}$ and $\sqrt{m+n} \sqrt{2 r} \sqrt{h_{k}} \leq \delta$. The claim of the lemma follows with $\lambda^{h}=\lambda^{\ell}, F_{1}^{h}=F_{1}^{\ell}$ and $F_{2}^{h}=F_{2}^{\ell}$.

LEMMA 4.2. Let $\mathcal{G}\left(h_{0}, \infty\right) \subset \mathcal{G}\left(h_{1}, \infty\right) \subset \cdots \subset \mathcal{G}\left(h_{N-1}, \infty\right)$ be a sequence of nested grids with $h_{0}>\cdots>h_{N-1}$. Assume that $\left(\lambda_{\alpha}, F_{\alpha}\right) \in \mathcal{H}_{N},\left|F_{\alpha}\right|<r$ and that in addition $\bar{F}=\sum_{\alpha=1}^{N} \lambda_{\alpha} F_{\alpha} \in \mathcal{G}\left(h_{0}, r\right)$. Then there exist $\left(\lambda_{\alpha}^{h}, F_{\alpha}^{h}\right) \in \mathcal{H}_{N}$ such that $F_{\alpha}^{h} \in \mathcal{G}\left(h_{N-1}, r+\delta\left(h_{0}, \ldots, h_{N-1}\right)\right), \bar{F}=\sum_{\alpha=1}^{N} \lambda_{\alpha}^{h} F_{\alpha}^{h}$ and

$$
\left|\lambda_{\alpha}-\lambda_{\alpha}^{h}\right| \leq q\left(h_{0}, \ldots, h_{N-1}\right), \quad\left|F_{\alpha}-F_{\alpha}^{h}\right| \leq \delta\left(h_{0}, \ldots, h_{N-1}\right) .
$$

Proof. The idea is to construct the matrices $F_{\alpha}^{h}$ recursively, starting from the average $\bar{F}$ and going backwards in the definition of the condition $\mathcal{H}_{N}$, until we reach the original matrices $F_{\alpha}$. Using the definitions in section 2 there exist $\mu_{1}^{2}, \mu_{2}^{2}$ and $G_{1}^{2}, G_{2}^{2}$ such that

$$
\bar{F}=\mu_{1}^{2} G_{1}^{2}+\mu_{2}^{2} G_{2}^{2} .
$$

We now apply Lemma 4.1. Since $\bar{F} \in \mathcal{G}\left(h_{0}, r\right)$ we can take $\delta$ arbitrarily small. In particular, if we choose $k=j$ in the proof of Lemma 4.1 (see (4.1)) we may fix $\mu_{1}^{2, h}, \mu_{2}^{2, h} \in(0,1)$ and $G_{1}^{2, h}, G_{2}^{2, h} \in \mathcal{G}\left(h_{1}, r\right)$ such that

$$
\begin{aligned}
\left|\mu_{i}^{2}-\mu_{i}^{2, h}\right| \leq \delta_{\lambda}=\frac{h_{1}}{h_{0}}=q_{0}, \\
\left|G_{i}^{2}-G_{i}^{2, h}\right| \leq \sqrt{m+n} \sqrt{2 r} \sqrt{h_{0}}+2 r \delta_{\lambda}=\delta_{0}+2 r q_{0},
\end{aligned}
$$


for $i=1,2$. Iterating this argument we obtain for $2 \leq \ell \leq N$ matrices $G_{i}^{\ell, h} \in$ $\mathcal{G}\left(h_{\ell-1}, r+\delta\left(h_{0}, \ldots, h_{\ell-1}\right)\right)$ and $\mu_{i}^{\ell, h} \in(0,1), 1 \leq i \leq \ell$, with the following properties:

$$
\begin{aligned}
\left|\mu_{i}^{\ell}-\mu_{i}^{\ell, h}\right| & \leq q_{\ell-2}, \\
\left|G_{i}^{\ell}-G_{i}^{\ell, h}\right| & \leq \delta_{\ell-3}+q_{\ell-3}+\sqrt{m+n} \sqrt{2 r} \sqrt{h_{\ell-2}}+2 r \frac{h_{\ell-1}}{h_{\ell-2}} \\
& =\sum_{p=1}^{\ell-2}\left(\delta_{p}+2 r q_{p}\right) .
\end{aligned}
$$

The claim of the lemma follows for $\ell=N$ since $F_{i}^{N}=G_{i}^{N}$.

LEMMA 4.3. Assume that $\left(\lambda_{\alpha}^{h}, F_{\alpha}^{h}\right) \in \mathcal{H}_{N}$ and that the matrices $F_{\alpha}^{h}$ and the associated matrices $H_{\alpha}^{h}$ (see section 2 for the definition) are contained in $\mathcal{G}(h, r)$. Let $\bar{F}^{h}=\sum_{\alpha=1}^{N} \lambda_{\alpha}^{h} F_{\alpha}^{h}$. Then $f^{h}=\mathcal{A}(f, \mathcal{G}(h, r))$ satisfies

$$
f^{h}\left(\bar{F}^{h}\right) \leq \sum_{\alpha=1}^{N} \lambda_{\alpha}^{h} f\left(F_{\alpha}^{h}\right) .
$$

Proof. This follows easily by induction using Remark 2.1. Note that the matrices $G_{1}, G_{2}$ defined in Remark 2.1 are contained in $\mathcal{G}(h, 2 r)$ if one splits the graph removing the edge containing the matrix $H_{N-1}$.

Proof of Theorem 3.2. Since $\mathcal{G}\left(h_{j}, r\right)$ contains only finitely many points it suffices to show that for $F^{h} \in \mathcal{G}\left(h_{j}, r\right)$ and $\varepsilon>0$ given there exists $k_{0} \in \mathbb{N}$ big enough such that

$$
f^{h_{i}}\left(F^{h}\right)-f^{r c}\left(F^{h}\right)<\varepsilon, \quad i \geq k_{0} .
$$

Let $M \geq \sup _{B_{2 r}(0)}|f|$. Using Lemma 2.4 we may choose $\left(\lambda_{\alpha}, F_{\alpha}\right) \in \mathcal{H}_{N}$ with $F_{\alpha} \in$ $B_{r}(0), F^{h}=\sum_{\alpha=1}^{N} \lambda_{\alpha} F_{\alpha}$ and

$$
f^{r c}\left(F^{h}\right) \geq \sum_{\alpha=1}^{N} \lambda_{\alpha} f\left(F_{\alpha}\right)-\frac{\tilde{\varepsilon}}{2} .
$$

Since $f$ is uniformly continuous on $\overline{B_{2 r}(0)}$ we may choose $\eta \in(0, r)$ such that $|F|$, $|G|<2 r,|F-G|<\eta$ implies $|f(F)-f(G)|<\varepsilon / 2$. Fix $K=\max \{2 N M, 6 r(N-1) \varepsilon / \eta\}$ and $h_{i_{0}}$ such that $\sqrt{m+n} \sqrt{2 r} \sqrt{h_{i_{0}}} \sqrt{K} /(\sqrt{K}-\sqrt{\varepsilon}) \leq \eta / 3$. Then choose $h_{i_{j}}$ for $j \geq 0$ such that $h_{i_{j+1}} / h_{i_{j}} \leq \varepsilon / K$. By definition, $q_{i_{j}} \leq \varepsilon / K$ and

$$
\begin{aligned}
\delta\left(h_{i_{0}}, \ldots, h_{i_{N-1}}\right) & =\sum_{j=0}^{N-2} \sqrt{m+n} \sqrt{2 r} \sqrt{h_{i_{j}}}+\sum_{j=0}^{N-2} 2 r \frac{\varepsilon}{K} \\
& =\sqrt{m+n} \sqrt{2 r} \sqrt{h_{i_{0}}} \sum_{j=0}^{N-2}\left(\frac{\varepsilon}{K}\right)^{j / 2}+2 r(N-1) \frac{\varepsilon}{K} \\
& =\sqrt{m+n} \sqrt{2 r} \sqrt{h_{i_{0}}} \frac{\sqrt{K}}{\sqrt{K}-\sqrt{\varepsilon}}+2 r(N-1) \frac{\varepsilon}{K} \leq \frac{2}{3} \eta .
\end{aligned}
$$

It follows from Lemma 4.2 and Lemma 4.3 that for $k \geq i_{N-1}$

$$
f^{h_{k}}\left(F^{h}\right) \leq \sum_{\alpha=1}^{N} \lambda_{\alpha}^{h} f\left(F_{\alpha}^{h}\right)
$$




$$
\begin{aligned}
& =\sum_{\alpha=1}^{N} \lambda_{\alpha} f\left(F_{\alpha}\right)+\sum_{\alpha=1}^{N}\left(\lambda_{\alpha}^{h}-\lambda_{\alpha}\right) f\left(F_{\alpha}\right)+\sum_{\alpha=1}^{N} \lambda_{\alpha}^{h}\left(f\left(F_{\alpha}^{h}\right)-f\left(F_{\alpha}\right)\right) \\
& \leq \sum_{\alpha=1}^{N} \lambda_{\alpha} f\left(F_{\alpha}\right)+\sum_{\alpha=1}^{N} \frac{\varepsilon}{2 N M}\|f\|_{\infty ; B_{2 r}(0)}+\sum_{\alpha=1}^{N} \lambda_{\alpha}^{h} \frac{\varepsilon}{2} \\
& \leq f^{r c}\left(F^{h}\right)+\varepsilon .
\end{aligned}
$$

If $f \in \mathcal{E}^{N}\left(B_{r}(0)\right)$, then there exists for all matrices $F \in \mathcal{G}\left(h_{k_{0}}, r\right)$ a family of pairs $\left(\lambda_{\alpha}, F_{\alpha}\right) \in \mathcal{H}_{N}, F_{\alpha} \in B_{r}(0)$ such that $f^{r c}(F)=\sum_{\alpha=1}^{N} \lambda_{\alpha} f\left(F_{\alpha}\right)$. For any choice of indices $k_{0} \leq i_{0}<\cdots<i_{N-1} \leq \ell$ we may construct $\left(\lambda_{\alpha}^{h}, F_{\alpha}^{h}\right) \in \mathcal{H}_{N}$ such that the estimates in Lemma 4.2 hold. Then

$$
\begin{aligned}
\left|f^{h_{\ell}}(F)-f^{r c}(F)\right| & \leq \sum_{\alpha=1}^{N}\left|\lambda_{\alpha}\right|\left|f\left(F_{\alpha}^{h}\right)-f\left(F_{\alpha}\right)\right|+\sum_{\alpha=1}^{N}\left|\lambda_{\alpha}-\lambda_{\alpha}^{h}\right|\left|f\left(F_{\alpha}^{h}\right)\right| \\
& \leq L \delta\left(h_{i_{0}}, \ldots, h_{i_{N-1}}\right)+\sup _{B_{2 r}(0)}|f| q_{N-2} .
\end{aligned}
$$

This proves the theorem.

5. Numerical experiments. In this section we briefly describe the implementation of the algorithm and discuss numerical examples. We used a uniform grid in the cube $[-a, a]^{4} \subset \mathbb{M}^{2 \times 2}$ with up to 67 points on each of the coordinate axes and the following sets of rank-one directions,

$$
\mathcal{R}_{h, k}=\left\{h R: R=a \otimes b, a \in \mathbb{Z}^{m}, b \in \mathbb{Z}^{n},\left|a_{i}\right|,\left|b_{i}\right| \leq k\right\}
$$

for $k=1,2,3$. One obtains 16,64 , and 256 directions, respectively. In our implementation we used a lexicographic ordering of the points on the grid and the rank-one directions. The discrete function $f^{h}$ defined on $\mathcal{G}(h, r)$ was stored as a one-dimensional vector, which was updated after each one-dimensional convexification. These can be done with $\mathcal{O}(n)$ operations if $n$ is the number of points on $\ell_{h, r}(F, R)$. The following method $[\mathrm{K}]$ uses the fact that $g^{h}:=f^{h}(F, R)$ is not convex at some point $x_{i}$ if

$$
\left\langle\left(\begin{array}{c}
x_{i-1}-x_{i} \\
g^{h}\left(x_{i-1}\right)-g^{h}\left(x_{i}\right)
\end{array}\right),\left(\begin{array}{rr}
0 & -1 \\
1 & 0
\end{array}\right)\left(\begin{array}{c}
x_{i+1}-x_{i} \\
g^{h}\left(x_{i+1}\right)-g^{h}\left(x_{i}\right)
\end{array}\right)\right\rangle<0 .
$$

The implementation uses a FIFO stack:

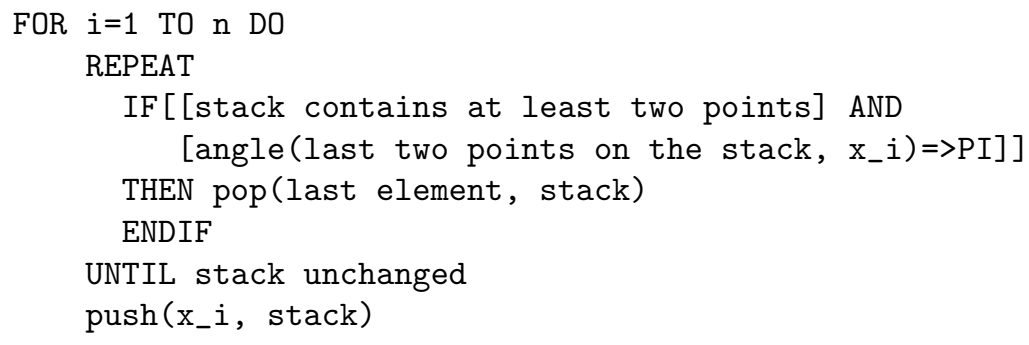

The stack contains at the end of the computation the points which lie on the convex envelope of the function. The affine interpolation yields the desired envelope of $g^{h}$. All computations were performed on a Silicon Graphics Origin 200 with four 180 MHZ R10k processors and 1GB memory at the Max Planck Institute for Mathematics in the Sciences, Leipzig. Our (strictly sequential) code needed, for example, 51 minutes 


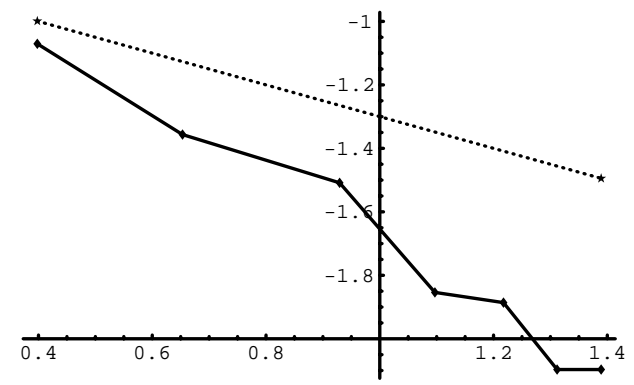

FIG. 5.1. The logarithm of the $L^{\infty}$-norm versus the logarithm of the width of the grid for the modified Kohn-Strang example. The dotted line has slope 1. (The logarithms are with respect to the base 10.)

for a loop over all matrices and all rank-one directions with $m=n=2, k=2$, and 33 points on the one-dimensional axes. Approximately 75 million one-dimensional convexifications were performed. Clearly all rank-one lines were considered more than once during this cycle. However, bookkeeping seems to be more time and memory consuming than allowing for these additional convexifications.

5.1. The Kohn-Strang example. Our first example arises in the context of homogenization and has been studied in detail in [KS1], [KS2]. Let

$$
W(\xi)=\left\{\begin{array}{cc}
1+|\xi|^{2}, & \xi \neq 0 \\
0, & \xi=0 .
\end{array}\right.
$$

The rank-one convex envelope can be computed explicitly (see [KS2, Section 5C]) and is given by

$$
W^{r c}(\xi)=\left\{\begin{array}{cc}
1+|\xi|^{2}, & \varrho(\xi) \geq 1, \\
2 \varrho-2 D, & \varrho(\xi) \leq 1,
\end{array}\right.
$$

where $D=|\operatorname{det}(\xi)|$ and $\varrho=\sqrt{|\xi|^{2}+2 D}$. Since $W$ does not satisfy the assumptions in Theorem 2.2 ( $W$ is not continuous at zero) we define the following modification:

$$
\tilde{W}(\xi)= \begin{cases}1+|\xi|^{2} & \text { if }|\xi| \geq \sqrt{2}-1 \\ 2 \sqrt{2}|\xi| & \text { if }|\xi| \leq \sqrt{2}-1\end{cases}
$$

Then $W^{r c} \leq \tilde{W} \leq W$ and therefore $\tilde{W}^{r c}=W^{r c}$. The proof in [KS2] shows that $W$ and hence $\tilde{W}$ belong to $\mathcal{E}^{2}$ and Theorem 3.2 guarantees the convergence of the algorithm. Figure 5.1 shows the $L^{\infty}$-norm of $f^{h_{i}}-f^{r c}$ on a uniform grid with $h_{i}=$ $2 / N_{i}, N_{i} \in\{5,9,17,25,33,41,49\}, \mathcal{R}_{h}=\mathcal{R}_{h, 3}$, and $\varepsilon=10^{-5}$ in the algorithm. The empirical convergence rate (with the error computed on the full grid, not just on a subgrid as in Theorem 3.2; it is impossible to get reasonable data on a sequence of properly nested grids) seems to be superlinear and thus much better than predicted.

5.2. A function related to the four gradient problem. The following example has been studied by different authors independently (see $[\mathrm{AH}],[\mathrm{CT}],[\mathrm{T}]$ ). Let

$$
\tilde{K}=\{(1,2),(2,-1),(-1,-2),(-2,1)\}=\left\{p_{1}, p_{2}, p_{3}, p_{4}\right\},
$$


and define $q_{1}=(1,-1), q_{2}=(-1,-1), q_{3}=(-1,1)$, and $q_{4}=(1,1)$. It follows from the references above

$$
\tilde{K}^{s c}=Q_{\infty}(0,1) \cup \bigcup_{i=1}^{4}\left[p_{i}, q_{i}\right],
$$

where $[a, b]=\{\lambda a+(1-\lambda) b: \lambda \in[0,1]\}$ and $Q_{\infty}=\{(x, y):|x|,|y| \leq 1\}$. Following [BFJK] we extend this example to $\mathbb{M}^{2 \times 2}$ by defining $K=\{\operatorname{diag}(s, t),(s, t) \in \tilde{K}\}$. This set is of particular interest, since despite the fact that $K$ does not contain any rank-one direction, $K^{r c}$ is nontrivial. Indeed $K^{r c}=\left\{\operatorname{diag}(s, t),(s, t) \in \tilde{K}^{s c}\right\}$. Since rank-one convexity implies separate convexity, the inclusion " $\supset$ " is clear; the other follows, for example, from the following proposition, Proposition 5.1. Let

$$
d(z)=\min \left\{\left|p_{i}-z\right|_{1}: i=1, \ldots, 4\right\}=\operatorname{dist}_{1}(z, K),
$$

where $|z|_{1}=|(x, y)|_{1}=|x|+|y|$ is the $\ell^{1}$-norm in $\mathbb{R}^{2}$. Define the rectangle $P_{1}=$ $[1,2] \times[-1,2]$ and the sector $S_{1}=\{x \geq 2,-x \leq y \leq x\}$ as well as the rotated rectangles and sectors, $P_{i}=R\left(\frac{i-1}{2} \pi\right) P_{1}$ and $S_{i}=R\left(\frac{i-1}{2} \pi\right) S_{1}$. Here $R(\varphi)$ denotes the rotation of the plane about zero with angle $\varphi$.

Proposition 5.1. Let $\Phi: \mathbb{M}^{2 \times 2} \rightarrow \mathbb{R}$ be given by

$$
\Phi(X)=d\left(X_{11}, X_{22}\right)+\frac{1}{6}\left(X_{12}^{2}+X_{21}^{2}\right) .
$$

Then

$$
\Phi^{r c}(X)=d^{s c}\left(X_{11}, X_{22}\right)+\frac{1}{6}\left(X_{12}^{2}+X_{21}^{2}\right)
$$

Moreover, if

$$
p(z)=\frac{1}{3}(x-1)(y+1)
$$

and

$$
h(z)= \begin{cases}x-y-3 & \text { if } y \leq-1, \\ x+\frac{1}{3}(y+1)-2 & \text { if } y \in[-1,2], \\ x+y-3 & \text { if } y \geq 2,\end{cases}
$$

then

$$
d^{s c}= \begin{cases}0 & \text { if } z \in Q_{\infty}(0,1), \\ p\left(R\left(-\frac{i-1}{2} \pi\right) z\right) & \text { if } z \in P_{i}, \\ h\left(R\left(-\frac{i-1}{2} \pi\right) z\right) & \text { if } z \in S_{i} .\end{cases}
$$

Proof. It is easy to see that $\Phi^{r c}(X) \leq d^{s c}\left(X_{11}, X_{22}\right)+\frac{1}{6}\left(X_{12}^{2}+X_{21}^{2}\right)=: \Psi(X)$. A short calculation shows that $\Psi$ is rank-one convex and thus the assertion of the proposition follows since $\Phi^{r c}$ is the largest rank-one convex minorant of $\Phi$. The formula for $d^{s c}$ is obtained by considering $d$ restricted to segments $\{s\} \times[-s, s]$, $s \geq 1$ and using the high degree of symmetry in the definition of $K$.

We computed $\Phi^{r c}$ on a uniform grid in $[-4,4]^{4}$. This example demonstrates the sensitive dependence of the quality of the approximation on the position of the grid. If $K$ is contained in the grid, then the $L^{\infty}$-error is immediately of order $10^{-5}$, while we observe only linear convergence if $K$ is not a subset of the grid points; see Figure 5.2 and Table 5.1. The first case corresponds to 17,33 , and 65 points on the coordinate axes, while the latter corresponds to computations with 19, 35, and 67 points, respectively. 


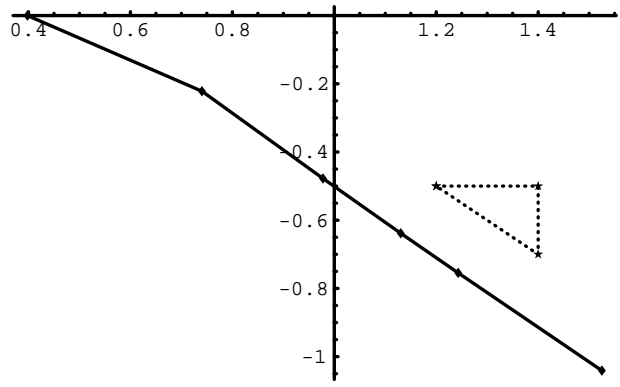

FIG. 5.2. The logarithm of the $L^{\infty}$-norm versus the logarithm of the width of the grid for the four gradient problem on general meshes which do not contain the zero set of $\Phi$. The dotted triangle has slope 1. (The logarithms are with respect to the base 10.)

TABLE 5.1

$L^{\infty}$-norm of the error in computations for the four gradient problem. The tabel shows the number of grid points on the coordinate axes and the error after one, two and three loops in the algorithm, respectively. Here a loop means a cycle over all matrices and all rank-one directions. We used $\mathcal{R}_{h}=\mathcal{R}_{h, 2}$.

\begin{tabular}{|c|c|c|c|}
\hline Gridpoints & Iteration 1 & Iteration 2 & Iteration 3 \\
\hline \hline 17 & 0.2222222 & 0.0034294 & 0.0000423 \\
19 & 0.4665883 & 0.3359462 & 0.3333656 \\
\hline 33 & 0.2222222 & 0.0037723 & 0.0000466 \\
35 & 0.3525501 & 0.1797479 & 0.1765110 \\
\hline 65 & 0.2222222 & 0.0039438 & 0.0000487 \\
67 & 0.2877217 & 0.0909544 & 0.0909113 \\
\hline
\end{tabular}

5.3. Does rank-one convexity imply quasiconvexity? This is still a fundamental open problem in the calculus of variations if the dimension $m$ of the target is 2. A counterexample for $m \geq 3$ has been given by Šverák in [Sv2]. Our rigorous convergence proof justifies the following test for $m \geq 2$. Guess, for example, on a triangulation of the unit cube $Q$ a piecewise affine test function which vanishes on $\partial Q$. Define $K=\{\nabla f(x): x \in Q\}$ and

$$
f(X)= \begin{cases}0 & \text { if } X \in K \\ \infty & \text { else }\end{cases}
$$

It is clear that $f^{q c}(0)=0$. If the algorithm shows that the numerical approximation of $f^{r c}$ in zero is positive, this could give some evidence that rank-one convexity does not imply quasiconvexity. Unfortunately, we did not discover any promising examples in $(2 \times 2)$-matrices. However, we found the following set of eighteen gradients in $(3 \times 2)$-matrices which could lead to a new counter example for $m=3$ :

$$
\begin{gathered}
\left(\begin{array}{rr}
0 & -2 \\
0 & 1 \\
-1 & 1
\end{array}\right),\left(\begin{array}{rr}
-2 & 0 \\
1 & 0 \\
1 & -1
\end{array}\right),\left(\begin{array}{rr}
-2 & 1 \\
0 & 0 \\
0 & 1
\end{array}\right),\left(\begin{array}{rr}
1 & -2 \\
-1 & 1 \\
0 & 1
\end{array}\right),\left(\begin{array}{rr}
2 & 0 \\
0 & 0 \\
1 & -1
\end{array}\right),\left(\begin{array}{rr}
1 & 1 \\
0 & 0 \\
-1 & 1
\end{array}\right), \\
\left(\begin{array}{rr}
-2 & 0 \\
1 & 1 \\
1 & -1
\end{array}\right),\left(\begin{array}{rr}
-2 & 0 \\
2 & 0 \\
1 & -1
\end{array}\right),\left(\begin{array}{rr}
1 & 1 \\
-1 & 2 \\
0 & -2
\end{array}\right),\left(\begin{array}{rr}
2 & 0 \\
0 & 1 \\
-1 & -1
\end{array}\right),\left(\begin{array}{rr}
1 & 0 \\
0 & 0 \\
-1 & -1
\end{array}\right),\left(\begin{array}{rr}
0 & 1 \\
-2 & 2 \\
0 & -2
\end{array}\right), \\
\left(\begin{array}{rr}
-2 & 2 \\
2 & -2 \\
1 & 0
\end{array}\right),\left(\begin{array}{rr}
0 & 0 \\
0 & 0 \\
-1 & 2
\end{array}\right),\left(\begin{array}{rr}
2 & -2 \\
0 & -2 \\
-1 & 1
\end{array}\right),\left(\begin{array}{rr}
-2 & 2 \\
0 & -2 \\
0 & 0
\end{array}\right),\left(\begin{array}{rr}
0 & 0 \\
-2 & 0 \\
0 & 2
\end{array}\right),\left(\begin{array}{rr}
2 & -2 \\
0 & -2 \\
1 & 1
\end{array}\right) .
\end{gathered}
$$


Question: Is $0 \in K^{r c}$ ? If not, then the rank-one convexification of the distance function to the set of these matrices is rank-one convex, but not quasiconvex.

Acknowledgments. Most of the work was done while the author held a postdoctoral research fellowship at the Center for Nonlinear Analysis at Carnegie Mellon University, Pittsburgh, whose hospitality and stimulating atmosphere are gratefully acknowledged. It is my pleasure to thank N. J. Walkington for fruitful discussion on the subject. I also thank the referees for their insightful comments.

\section{REFERENCES}

[AH] R. Aumann And S. HaRt, Bi-convexity and bi-martingales, Israel J. Math., 54 (1986), pp. 159-180.

[BJ] J. M. Ball And R. D. James, Proposed experimental tests of a theory of fine microstructure and the two-well problem, Philos. Trans. Roy. Soc. London Ser. A, 338 (1992), pp. 389450.

[BFJK] K. Bhattacharya, N. Firoozye, R. D. James, and R. V. Kohn, Restrictions on microstructure, Proc. Roy. Soc. Edinburgh Sect. A, 124 (1994), pp. 843-878.

[B] Y. Brenier, Un algorithme rapide pour le calcul de transformées de Legendre-Fenchel, C. R. Acad. Sci. Paris Sér. I Math., 308 (1989), pp. 587-589.

[CT] E. Casadio-TARABUSi, An algebraic characterization of quasiconvex functions, Ricerche Mat., 42 (1993), pp. 11-24.

[Co] L. Corrias, Fast Legendre-Fenchel transform and applications to Hamilton-Jacobi equation and conservation laws, SIAM J. Numer. Anal., 33 (1996), pp. 1534-1558.

[Da] B. Dacorogna, Direct Methods in the Calculus of Variations, Springer, New York, 1989.

[DM] B. Dacorogna And P. Marcellini, Sur le problème de Cauchy-Dirichlet pour les systèmes d'équations non linéaires du premier ordre, C. R. Acad. Sci. Paris Sér. I Math., 323 (1996), pp. 599-602.

[DW] G. Dolzmann and N. J. Walkington, Numer. Math., accepted.

[KS1] R. V. Kohn And G. Strang, Optimal design and relaxation of variational problems I, Comm. Pure Appl. Math., 39 (1986), pp. 113-137.

[KS2] R. V. Kohn And G. Strang, Optimal design and relaxation of variational problems II, Comm. Pure Appl. Math., 39 (1986), pp. 139-182.

[K] U. KORTENKAMP, personal communication.

[LDR] H. LE DRET AND A. RAOult, The quasiconvex envelope of the Saint Venant-Kirchhoff stored energy function, Proc. Roy. Soc. Edinburgh, 125 (1995), pp. 1179-1192.

[L] M. Luskin, On the computation of crystalline microstructure, Acta Numerica, 5 (1996), pp. 191-258

[MP] J. MATOUŠEK AND P. Plecháč, On functional separately convex hulls, Discrete Comput. Geom., 19 (1998), pp. 105-130.

[M] C. B. Morrey, Multiple Integrals in the Calculus of Variations, Springer, New York, 1966.

[MS] S. MülLER AND V. ŠVERÁK, Attainment results for the two-well problem by convex integration, in Geometric Analysis and the Calculus of Variations, J. Jost, ed., International Press, Cambridge, MA, 1996, pp. 239-251.

[Pe1] P. Pedregal, Laminates and microstructure, European J. Appl. Math., 4 (1993), pp. 121149.

[Pe2] P. Pedregal, The concept of a laminate, in Microstructure and Phase Transition, D. Kinderlehrer, ed., IMA Vol. Math. Appl. 54, Springer, New York, 1993, pp. 129-142.

[Pi] A. C. Pipkin, Elastic materials with two preferred sates, Quart. J. Mech. Appl. Math., 44 (1991), pp. 1-15.

[Pl] P. PleChÁČ, An iterative algorithm for rank-1 convexification, in preparation.

[Sv1] V. Šverák, Examples of rank-one convex functions, Proc. Roy. Soc. Edinburgh Sect. A, 114 (1990), pp. 237-242.

[Sv2] V. ŠverÁk, Rank-one convexity does not imply quasiconvexity, Proc. Roy. Soc. Edinburgh Sect. A, 120 (1992), pp. 185-189.

[Sv3] V. ŠverÁK, On the problem of two wells, in Microstructure and Phase Transition, D. Kinderlehrer, ed., IMA Vol. Math. Appl. 54, Springer, New York, 1993, pp. 183-189.

[T] L.TARTAR, Some remarks on separately convex functions, in Microstructure and Phase 
Transition, D. Kinderlehrer, ed., IMA Vol. Math. Appl. 54, Springer, New York, 1993, pp. 191-204.

[Z] K. ZHANG, On various semiconvex hulls in the calculus of variations, Calc. Var. Partial Differential Equations, 6 (1998), pp. 143-160. 